\title{
Periodic structures stiffness topology optimization
}

\section{João Victor Watanabe Nunes, Renato Pavanello.}

\begin{abstract}
Stiffness topology optimization aims to determine the best material arrangement, capable of conciliating high structural stiffness and low weight, which handles certain loading conditions subjected to predefined boundary conditions. The imposed periodic constrain is fundamented on the fact that structures made of periodic materials behave as a homogeneous continuum, because the macro-structure cells are modeled as a uniform medium composed by periodic material. The optimization algorithm used in this work is the BESO (Bi-directional Evolutionary Structural Optimization), which analyzes the structure, under its loadings and boundary conditions, and generates the optimum topology through addition and removal of material. However, in order to reduce the high computational costs of this method when applied to very refined meshes, which is the case with most periodic structures, emphasis was placed on the integration between Matlab and Ansys softwares, with promising results.
\end{abstract}

\section{Key words:}

Topology optimization, periodic structures, BESO.

\section{Introduction}

Periodic structures are composed by a microstructure that repeats, periodically, through the material. A well known example of periodic materials are honeycomb structures. These structures can have excellent properties, such as high stiffness and low weight, due to a topology optimization process. The optimization process aims to find the best topology (material distribution) of a structure, so it can improve material performance given the boundary and loading conditions. The present work has two main objectives: optimize periodic material stiffness, by minimizing structure mean compliance; and reduce the implementation costs of the proposed optimization algorithm, by integrating the softwares Matlab and Ansys.

\section{Results and Discussion}

The topology obtained, for the well-known case of "short cantilever beam", using the implemented algorithm, with the following main parameters: cantilever beam constrained at the left end and $100 \mathrm{~N}$ downward distributed load applied at the right end, beam dimensions: $32 \mathrm{~m}$ length by $20 \mathrm{~m}$ height with a $2 \mathrm{~m}$ nondesign domain at the top and bottom end of the beam; is shown below:

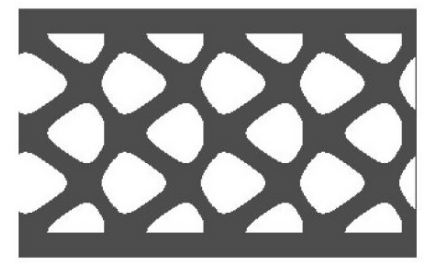

Image 1. Short cantilever beam topology.

The design domain was divided into a 4 by 2 unit cells to form a periodic structure and the mesh is made of 64200 elements.

The developed algorithm, integrating Matlab and Ansys softwares, proved to be more efficient than the conventional algorithm, using Matlab only, from a mesh size of 70000 elements, as shown below:

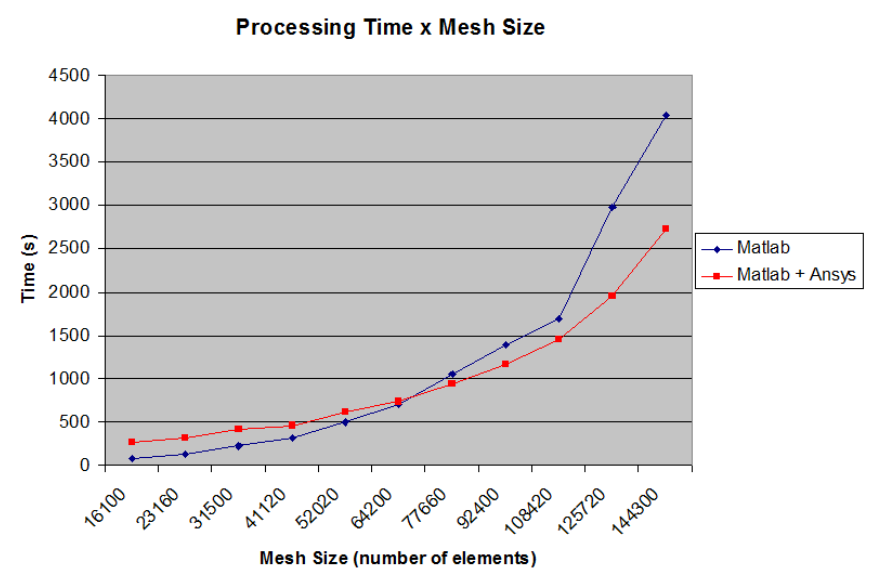

Image 2. Comparative graph between conventional algorithm and integrated algorithm.

Running a mesh of 144300 elements the reduction of execution time is around $32 \%$ and, from the graph, one can infer that the reduction of execution time can be greater the larger the mesh size.

\section{Conclusions}

From the above it can be concluded that the algorithm developed is a reliable, robust and efficient tool for topology optimization of periodic structures. The main goals were successfully accomplished and the advantages of the integrated algorithm are several, as it is more efficient for large mesh problems: periodic structures and 3D cases, for example.

\section{Acknowledgement}

I am grateful to CNPq for its financial support.

\footnotetext{
${ }^{1}$ Huang, X.; Xie, Y. M. Evolutionary topology optimization of continuum structures: methods and applications. 2010.
}

${ }^{2}$ Moaveni, S. Finite element analysis theory and application with Ansys. 1999. 